\title{
Synthesis and in vitro Antiproliferative Activity of Flavone and 6-Hydroxyflavone Oxime Ethers Derivatives
}

\author{
John E. Díaz, ${ }^{a}$ Diana C. Martinez, ${ }^{a}$ Lina V. López, ${ }^{a}$ Gina M. Mendez, ${ }^{b}$ Ricardo Vera ${ }^{a}$ and \\ Alix E. Loaiza*,a
}

${ }^{a}$ Departamento de Química, Facultad de Ciencias, Pontificia Universidad Javeriana, Cra. 7 No. 40-62, Edificio Carlos Ortiz, 110231561 Bogotá, Colombia

${ }^{b}$ Facultad de Medicina, Universidad de Ciencias Aplicadas y Ambientales, Calle 222 No. 55-37, 111166003 Bogotá, Colombia

\begin{abstract}
Herein we report the synthesis of a series of $O$-alkyl oximes of flavone and 6-hydroxyflavone using a simple experimental protocol under solvent free conditions with yields up to $87 \%$. Cytotoxicity of all compounds was evaluated against MDA-MB-231, PC-3, A-549 and MRC-5 cells. $\mathrm{IC}_{50}$ values for two compounds were determined to be in the range 28.7-47.8 $\mu \mathrm{M}$ against all tested cell lines. Oxime ethers derivatives showed $\mathrm{IC}_{50}$ values between 28.7 and $49.5 \mu \mathrm{M}$ against MDA-MB-231, while the best activity was obtained for 6-hydroxyflavone with an $\mathrm{IC}_{50}$ of $3.4 \mu \mathrm{M}$ against this cell line. Compounds containing the substituent hydroxyl at the position six of flavone system displayed the best antiproliferative activity over MDA-MB-231 cells, being necessary this group to improve the sensibility on this type of cells. The antiproliferative activity of 6-hydroxyflavone is drastically diminished when the carbonyl group of flavone is changed by an oxime ether.
\end{abstract}

Keywords: synthesis, oxime ethers, flavone, 6-hydroxyflavone, antiproliferative activity

\section{Introduction}

Oxime ethers are useful and versatile compounds in organic synthesis, they are commonly easily prepared and relatively stable to moisture; thus, they can be stored under air during long periods. ${ }^{1}$ Oxime is a pharmacophore group present in a variety of compounds with diverse biological activities, for instance, some $\alpha, \beta$-unsaturated oximes are known as anticancer, ${ }^{2}$ immunosuppressive, ${ }^{3}$ and antibacterial ${ }^{4}$ agents.

On the other hand, flavone scaffold is an important core in many compounds displaying a variety of pharmacological properties that depend on their substitution patterns. The wide range of biological activities of flavones has attracted great interest in the synthesis of derivatives with the objective of developing new therapeutic agents. ${ }^{5}$

Although oxime ether derivatives of flavones could display relevant biological activity, there are only a few reports about these kind of compounds, suggesting that these derivatives are not as promptly prepared. The first synthesis of a flavone oxime was reported in 1952 using

*e-mail: aloaiza@javeriana.edu.co hydroxylamine, and 4-thionflavone or 4-thionflavone methiodide as precursos. ${ }^{6}$ Previous attempts using directly hydroxylamine and a flavone conduced to 3-o-hydroxyphenyl-5-phenylisooxazole instead of the oxime. $^{7}$

Meshcheryakova et ll $^{8}$ reported the synthesis of some flavone oxime ether derivatives using 4,4-dichloroflavene or 4-thionflavone and $O$-alkyl hydroxylamines, and the evaluation of their pharmacological activity revealed that they act on the central nervous system. Green et al. ${ }^{9}$ achieved the synthesis of 2-arylchromenone oximes using Ollis or Lawesson methods; and patented their use as inhibitors of protein kinases.

Schann et al. ${ }^{10}$ synthesized 2-heteroarylchromenone oximes as allosteric modulators of metabotropic glutamate receptors, via the synthesis of $O$ - $t$-butyl oxime ethers employing microwave (MW) irradiation in methanol, and further deprotection. They also prepared some of these compounds directly with hydroxylamine under MW, with poor yields.

Therefore, this work was undertaken with the aim of extending the knowledge about the synthesis, characterization and applications of flavone oxime ethers. 
We prepared and evaluated the antiproliferative activity of twelve derivatives containing $O$-alkyl, $O$-benzyl and $O$-allyl moieties.

\section{Results and Discussion}

\section{Chemistry}

In order to obtain the target compounds by a previously optimized protocol, we carried out experiments using the model reaction between flavone (1a) and $O$-methylhydroxylamine hydrochloride (2a) in pyridine as a solvent and a base (Table 1, entry 1 ). Under these conditions, no evidence of the formation of compound $\mathbf{3 a}$ was observed after $18 \mathrm{~h}$, and the flavone was mostly recovered.

Then, we adapted procedures reported in the literature for analog structures using microwave irradiation. The reaction with $O$-methylhydroxylamine hydrochloride in methanol under microwave for 30 minutes (entry 2$)^{10} \mathrm{did}$ not give rise to compound $\mathbf{3 a}$; thus, we decided to perform a new attempt using the former conditions, adding pyridine as the base (entry 3 ). This procedure allowed us obtaining the desired oxime ether $\mathbf{3 a}$ in a $19 \%$ according to ${ }^{1} \mathrm{H}$ nuclear magnetic resonance (NMR) spectra of the crude mixture, as a single isomer.

Due to the low reactivity displayed by flavone, we employed the conditions reported by Meshcheryakova et al., ${ }^{8}$ that includes the transformation of flavone in 4,4-dichloroflavene (entry 4). The reaction was monitored by gas chromatography-mass spectrometry (GC-MS) and the dichloride intermediate was detected after the first step (a), but no evidence of the formation of 3a was observed after the second one (b).
A fifth experiment (entry 5), employing 6 equivalents of both $O$-methylhydroxylamine hydrochloride and dry pyridine at $140{ }^{\circ} \mathrm{C}$ was carried out in order to displace the equilibrium towards the formation of oxime ether $\mathbf{3 a}$. After $2 \mathrm{~h}$ under these conditions, the starting material was not completely consumed, but the desired oxime ether was observed in $75 \%$ by ${ }^{1} \mathrm{H}$ NMR.

Guided by the previous result, and with the aim of finding the molar ratio of the reagents that allowed to obtain the highest yield of $\mathbf{3 a}$, we performed several experiments varying the equivalents of both $\mathbf{2 a}$ and pyridine. Finally we found that conditions shown in entry 6 , afforded the maximum amount of product ( $75 \%$ calculated by ${ }^{1} \mathrm{H}$ NMR, $54 \%$ isolated), with the lowest equivalents of starting materials.

Based on the results shown above, we synthesized a series of oxime ethers of flavone and 6-hydroxyflavone (3b-l) using the experimental conditions of entry 6. In order to synthesize oxime ethers $\mathbf{3 c - f}, \mathbf{i}-\mathbf{l}$, it was necessary to obtain the $O$-allyl and $O$-benzylhydroxylamines $\mathbf{5 c}$-f no commercially available, via Gabriel synthesis, and their hydrochlorides 2c-f (Scheme 1).

The E/Z geometries of oxime ethers $\mathbf{3 a - l}$, were determined by 2D NOESY (nuclear Overhauser effect spectroscopy) experiments, which allowed to evidence correlations between hydrogens of $O-\mathrm{CH}_{2}$ group and the heterocycle ring, showing that the $E$ isomer was the most favored product.

\section{Antiproliferative activity}

Cytotoxicity of all compounds was evaluated against MDA-MB-231, PC-3, A-549 and MRC-5 cells. Flavone (1a)

Table 1. Evaluation of reaction conditions to obtain compound 3a

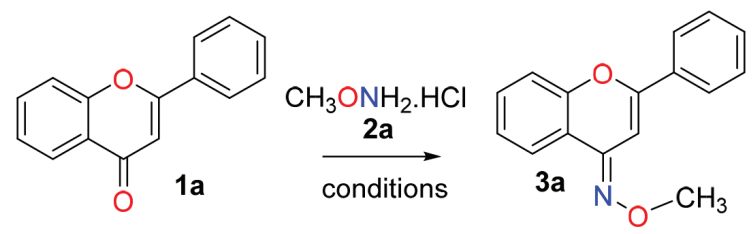

\begin{tabular}{|c|c|c|}
\hline entry & Conditions & $\mathbf{3} \mathbf{a}^{\mathrm{a}} / \%\left(\mathbf{3} \mathbf{a}^{\mathrm{b}} / \%\right)$ \\
\hline 1 & 2a (2.0 equiv.), Py, reflux, $18 \mathrm{~h}$ & 0 \\
\hline 2 & 2a (2.0 equiv.), $\mathrm{MeOH}, \mathrm{MW} 150 \mathrm{~W}, 30 \mathrm{~min}$ & 0 \\
\hline $3^{\mathrm{c}}$ & 2a (2.0 equiv.), Py (2.0 equiv.), $\mathrm{MeOH}, \mathrm{MW} 150 \mathrm{~W}, 30 \mathrm{~min}$ & $5(19)$ \\
\hline 4 & (a) $\mathrm{SOCl}_{2}, \mathrm{Et}_{3} \mathrm{~N}_{\text {(dry }}, \mathrm{CHCl}_{3 \text { (dry) }}$, reflux, $5 \mathrm{~h}$; (b) $2 \mathrm{a}$ (1.2 equiv.), $1.5 \mathrm{~h}$ & 0 \\
\hline 5 & $2 \mathrm{a}$ (6.0 equiv.), $\mathrm{Py}_{(\text {dry })}\left(6.0\right.$ equiv.), $140^{\circ} \mathrm{C}$ (oil bath), $2.5 \mathrm{~h}$ & (75) \\
\hline $6^{\mathrm{d}}$ & 2a (2.5 equiv.), $\mathrm{Py}_{\text {(dry) }}$ ( 3.5 equiv.), $140{ }^{\circ} \mathrm{C}$ (oil bath), $2 \mathrm{~h}$ & $54(75)$ \\
\hline
\end{tabular}

${ }^{\mathrm{a}}$ Isolated yield; ${ }^{\mathrm{b}}$ calculated yield by ${ }^{1} \mathrm{H}$ NMR; ${ }^{\mathrm{c}}$ method A; ${ }^{\mathrm{d}}$ method B. 


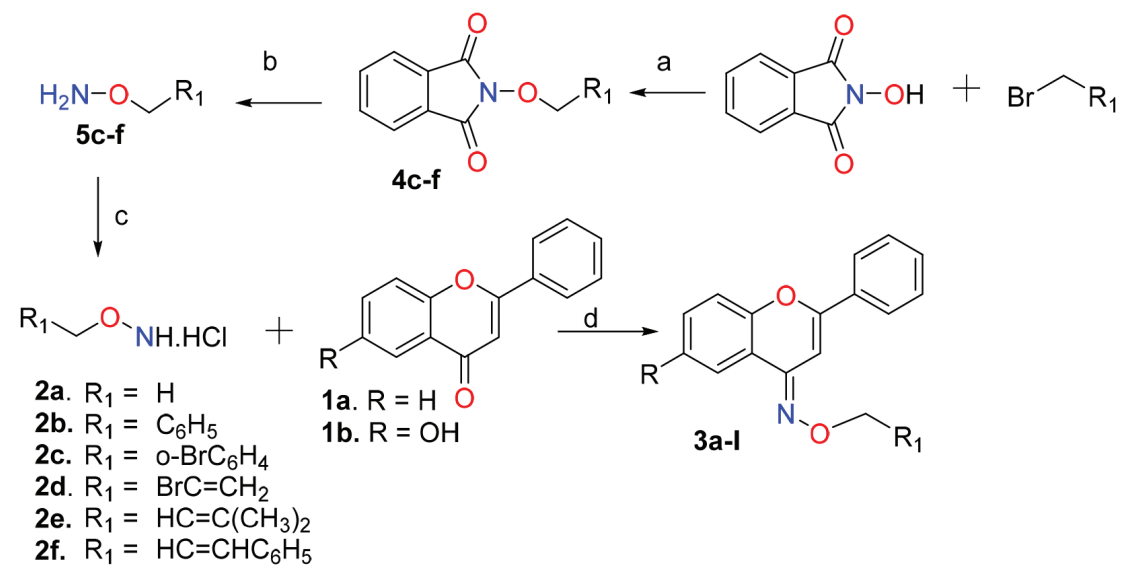

Scheme 1. (a) $\mathrm{K}_{2} \mathrm{CO}_{3}$, DMSO, room temperature, 2-3 h (87-97\%); (b) $\mathrm{NH}_{2} \mathrm{NH}_{2} \cdot \mathrm{H}_{2} \mathrm{SO}_{4}, \mathrm{~K}_{2} \mathrm{CO}_{3}, \mathrm{Et}_{2} \mathrm{O}, \mathrm{H}_{2} \mathrm{O}$, reflux, 5 h (76-90\%); (c) $\mathrm{HCl}(\mathrm{g}), \mathrm{Et} 2 \mathrm{O}$; (d) $\mathrm{Py} / \Delta(54-87 \%)$.

and its oxime derivatives (3a-f) showed no relevant activity at the evaluated concentrations. $\mathrm{IC}_{50}$ values for compounds 3h,i presenting a hydroxyl and a benzyl group, were determined to be in the range of $28.7-47.8 \mu \mathrm{M}$ for all tested cell lines. Compounds $3 g-1$ containing a hydroxyl group at sixth position, showed $\mathrm{IC}_{50}$ between 28.7-49.5 $\mu \mathrm{M}$ against MDA-MB-231; however, the best activity was obtained for 6-hydroxyflavone (1b) with an $\mathrm{IC}_{50}$ of $3.4 \mu \mathrm{M}$ against the same cell line (Table 2). Previous studies have shown that 6-hydroxyflavone also exhibits activity over the leukemia cell lines HL-60 $\left(\mathrm{IC}_{50}=2.8 \mu \mathrm{M}\right)$ and MOLT-4 $\left(\mathrm{IC}_{50}=6.3 \mu \mathrm{M}\right){ }^{11}{ }^{11}$ It is important to emphasize that no antiproliferative activity was displayed by compound $\mathbf{1 b}$ against healthy fibroblasts from lung (MRC-5).

On the other hand, the antitumor activity of baicalein, a naturally ocurring flavonoid used in Chinese herbal medicine, which contains hydroxyl groups at positions 5, 6 and 7, has motivated to develop studies aimed to verify the inhibitory effects and the mechanism involved in its antimetastatic effect against MDA-MB-231 cells. The $\mathrm{IC}_{50}$ value of baicalein $(59.5 \mu \mathrm{M}),{ }^{12}$ higher than the $\mathrm{IC}_{50}$ determined for 6-hydroxyflavone in this work over the same cell line, motivates our interest in continuing the study of this compound.

It is important to notice that despite the $\mathrm{IC}_{50}$ values found in this work are not comparable with the reference drug vincristine $\left(\mathrm{IC}_{50}=0.008 \mu \mathrm{M}\right.$ against MDA-MB-231), the search for novel cytotoxic agents with selectivity for a particular cell line, and the comprenhension of mechanisms of action, are necessary tasks towards the development of new drugs that allow to improve the existing anticancer therapies.

Table 2. In vitro $\mathrm{IC}_{50}$ of compounds $\mathbf{1}$ and $\mathbf{3}$ against MDA-MB-231, PC-3, A-549 and MRC-5 cells

\begin{tabular}{|c|c|c|c|c|c|c|}
\hline \multirow{2}{*}{ Compound } & \multirow{2}{*}{$\mathrm{R}$} & \multirow{2}{*}{$\mathrm{R}_{1}$} & \multicolumn{4}{|c|}{$\mathrm{IC}_{50} /(\mu \mathrm{mol} \mathrm{L}-1)$} \\
\hline & & & MDA-MB-231 & PC-3 & A-549 & MRC-5 \\
\hline $1 \mathbf{a}$ & $\mathrm{H}$ & - & $>50$ & $>50$ & $>50$ & $>50$ \\
\hline $1 \mathrm{~b}$ & $\mathrm{OH}$ & - & $3.4 \pm 0.1$ & $>50$ & $>50$ & $>50$ \\
\hline $3 \mathbf{a}$ & $\mathrm{H}$ & $\mathrm{CH}_{3}$ & $>50$ & $>50$ & $>50$ & $>50$ \\
\hline $3 \mathbf{b}$ & $\mathrm{H}$ & $\mathrm{CH}_{2} \mathrm{C}_{6} \mathrm{H}_{5}$ & $>50$ & $>50$ & $>50$ & $>50$ \\
\hline $3 \mathbf{c}$ & $\mathrm{H}$ & $\mathrm{CH}_{2}-\mathrm{o}-\mathrm{BrC}_{6} \mathrm{H}_{4}$ & $>50$ & $>50$ & $>50$ & $>50$ \\
\hline 3d & $\mathrm{H}$ & $\mathrm{CH}_{2} \mathrm{CBr}=\mathrm{CH}_{2}$ & $>50$ & $>50$ & $>50$ & $>50$ \\
\hline $3 e$ & $\mathrm{H}$ & $\mathrm{CH}_{2}-\mathrm{CH}=\mathrm{C}\left(\mathrm{CH}_{3}\right)_{2}$ & $>50$ & $>50$ & $>50$ & $>50$ \\
\hline $3 f$ & $\mathrm{H}$ & $\mathrm{CH}_{2}-\mathrm{CH}=\mathrm{CHC}_{6} \mathrm{H}_{5}$ & $>50$ & $>50$ & $>50$ & $>50$ \\
\hline $3 g$ & $\mathrm{OH}$ & $\mathrm{CH}_{3}$ & $49.5 \pm 0.8$ & $>50$ & $>50$ & $>50$ \\
\hline $3 \mathbf{h}$ & $\mathrm{OH}$ & $\mathrm{CH}_{2} \mathrm{C}_{6} \mathrm{H}_{5}$ & $28.7 \pm 2.7$ & $47.8 \pm 0.6$ & $40.2 \pm 0.5$ & $44.5 \pm 0.7$ \\
\hline $3 \mathbf{i}$ & $\mathrm{OH}$ & $\mathrm{CH}_{2}-\mathrm{O}-\mathrm{BrC}_{6} \mathrm{H}_{4}$ & $40.4 \pm 1.7$ & $43.6 \pm 2.6$ & $47.4 \pm 0.9$ & $39.8 \pm 2.7$ \\
\hline $3 \mathbf{j}$ & $\mathrm{OH}$ & $\mathrm{CH}_{2} \mathrm{CBr}=\mathrm{CH}_{2}$ & $34.8 \pm 2.2$ & $>50$ & $>50$ & $39.7 \pm 2.5$ \\
\hline $3 \mathbf{k}$ & $\mathrm{OH}$ & $\mathrm{CH}_{2}-\mathrm{CH}=\mathrm{C}\left(\mathrm{CH}_{3}\right)_{2}$ & $45.4 \pm 0.3$ & $>50$ & $>50$ & $>50$ \\
\hline 31 & $\mathrm{OH}$ & $\mathrm{CH}_{2}-\mathrm{CH}=\mathrm{CHC}_{6} \mathrm{H}_{5}$ & $38.7 \pm 3.4$ & $>50$ & $>50$ & $>50$ \\
\hline
\end{tabular}

The $\mathrm{IC}_{50}$ value was defined as the concentration of the compound which caused a $50 \%$ decrease of the cell viability. 


\section{Conclusions}

The synthesis of twelve flavone and 6-hydroxyflavone oxime ether derivatives was achieved with reasonably good yields as a single isomer $(E)$, using a simple experimental protocol. Changing the carbonyl group of flavone for an oxime ether did not increase the cytotoxic activity of the derivatives against the tested line cells. Hydroxyl group at the position six of flavone system is necessary to display activity against MDA-MB-231 since all compounds containing this substituent showed antiproliferative activity over this cell line. The antiproliferative activity of 6-hydroxyflavone is drastically diminished when the carbonyl group of flavone is changed by an oxime ether.

\section{Experimental}

\section{Chemistry}

${ }^{1} \mathrm{H}$ and ${ }^{13} \mathrm{C}$ NMR spectra were acquired on a Bruker Avance spectrometer (300 and $75 \mathrm{MHz}$, respectively) in $\mathrm{CDCl}_{3}$ or $\mathrm{CD}_{3} \mathrm{OD}$. Chemical shifts and configuration were assigned with the help of HSQC-edit (heteronuclear single quantum correlation), HMBC (heteronuclear multiple bond correlation), COSY (correlation spectroscopy) and NOESY experiments. High-resolution mass spectra (HRMS) were recorded on an Agilent 6520 q-TOF-MS instrument with orthogonal ESI (electrospray ionization). GC-MS analyses were performed on an Agilent 6850 series II gas chromatograph coupled to an Agilent 5975B VL mass spectrometer (electron ionization, $70 \mathrm{eV}$ ) equipped with split/splitess inlet (split relation $15: 1,260^{\circ} \mathrm{C}$ ), Agilent 6850 series automatic injector, and Agilent HP-5MS column $(30 \mathrm{~m} \times 0.25 \mathrm{~mm} \times 0.25 \mu \mathrm{m})$; initial oven temperature $80{ }^{\circ} \mathrm{C}$ for $1 \mathrm{~min}$, then a temperature ramp of $10^{\circ} \mathrm{C} \mathrm{min}^{-1}$ to $320^{\circ} \mathrm{C}$ (hold $3 \mathrm{~min}$ ); total run time $28 \mathrm{~min}$. Melting points were determined on a Thermo Fisher Scientific Rochford 9100 apparatus.

\section{Synthesis of oxime ether derivates 3a-I}

\section{Method A (entry 3)}

A solution of flavone 1a $(1.0 \mathrm{mmol}), O$-methyl hydroxylamine hydrochloride $(2.0 \mathrm{mmol})$, and pyridine $(2.0 \mathrm{mmol})$ in methanol $(6.6 \mathrm{~mL})$, was irradiated at $150 \mathrm{~W}$ for 30 minutes. The reaction mixture was treated with distilled water and extracted with dichloromethane. The organic phase was dried with $\mathrm{Na}_{2} \mathrm{SO}_{4}$, filtered and concentrated under reduced pressure. The crude mixture was purified by column chromatography using silica gel and hexane: $\mathrm{CH}_{2} \mathrm{Cl}_{2}(4: 1)$, yielding $5 \%$ of $\mathbf{3 a}$.
Method B (entry 6)

A mixture of flavone or 6-hydroxyflavone 1a-b $(1.0 \mathrm{mmol})$, an appropriate $O$-alkyl hydroxilamine hydrochloride (2a-e) $(2.5 \mathrm{mmol})$, and dry pyridine (3.5 mmol), was stirred at $140{ }^{\circ} \mathrm{C}$ for $2-4 \mathrm{~h}$. The reaction mixture was treated with distilled water and extracted with dichloromethane. The organic phase was dried with $\mathrm{Na}_{2} \mathrm{SO}_{4}$, filtered and concentrated under reduced pressure. The organic combined were purified by column chromatography (silica gel, hexane: $\mathrm{CH}_{2} \mathrm{Cl}_{2} 4: 1$ or $\mathrm{CH}_{2} \mathrm{Cl}_{2}$ ) to provide products $\mathbf{3 a}-\mathbf{I}$.

\section{2-Phenyl-4H-chromen-4-one $\mathrm{O}$-methyloxime (3a)}

Yield 54\%; mp 57-58 ${ }^{\circ} \mathrm{C}$; ${ }^{1} \mathrm{H}$ NMR $\left(300 \mathrm{MHz}, \mathrm{CDCl}_{3}\right)$ $\delta 4.05$ (s, 3H, $\left.\mathrm{OCH}_{3}\right), 7.08(\mathrm{~s}, 1 \mathrm{H}, \mathrm{C}=\mathrm{CH}), 7.30-7.32(\mathrm{~m}$, 2H, H-6, 7 Ar), 7.42-7.49 (m, 4H, H-8, H-3', 4', 5' Ar), 7.89-7.92 (m, 2H, H-2', 6' Ar), 8.04 (dd, 1H, J 8.1, $1.5 \mathrm{~Hz}$, $\mathrm{H}-5 \mathrm{Ar}) ;{ }^{13} \mathrm{C} \mathrm{NMR}\left(75 \mathrm{MHz}, \mathrm{CDCl}_{3}\right) \delta 62.06\left(\mathrm{OCH}_{3}\right), 93.81$ $(\mathrm{C}=\underline{\mathrm{CH}}), 117.53$ (C-6 Ar), 118.37 (C-4a Ar), 123.01 (C-5 Ar), 124.65 (C-7 Ar), 125.71 (C-2', 6' Ar), 128.65 (C-8 Ar), 130.25 (C-4' Ar), 130.43 (C-3', 5' Ar), 132.89 (C-1' Ar), $143.84(\mathrm{C}-8 \mathrm{a}$ Ar), $151.91(\mathrm{C}=\mathrm{N}), 155.23(\mathrm{C}=\mathrm{CH})$. Mass spectrum, $m / z$ (relative intensity $\left.\left(I_{\text {rel }}\right), \%\right): 251[\mathrm{M}]^{+}$ (72), 236 (15), 206 (100). Found, $m / z: 252.1025[\mathrm{M}+\mathrm{H}]^{+}$, $\mathrm{C}_{16} \mathrm{H}_{14} \mathrm{NO}_{2}$. Calculated, $m / z: 252.1025$.

\section{2-Phenyl-4H-chromen-4-one O-benzyloxime (3b)}

Yield 70\%; mp 69-70 ${ }^{\circ} \mathrm{C}$; ${ }^{1} \mathrm{H}$ NMR $\left(300 \mathrm{MHz}, \mathrm{CDCl}_{3}\right)$ $\delta 5.30\left(\mathrm{~s}, 2 \mathrm{H}, \mathrm{OCH}_{2}\right), 7.14(\mathrm{~s}, 1 \mathrm{H}, \mathrm{C}=\mathrm{CH}), 7.21-7.37(\mathrm{~m}$, 8H, H-6, 7, 8, 2", 3", 4", 5", 6" Ar), 7.39-7.52 (m, 3H, H-3', 4', 5' Ar), 7.87-7.91 (m, 2H, H-2', 6' Ar), 8.05 (dd, $1 \mathrm{H}, J 8.1,1.5 \mathrm{~Hz}, \mathrm{H}-5 \mathrm{Ar}) ;{ }^{13} \mathrm{C}$ NMR $\left(75 \mathrm{MHz}, \mathrm{CDCl}_{3}\right.$ ) $\delta 76.22\left(\mathrm{OCH}_{2}\right), 94.07(\mathrm{C}=\mathrm{CH}), 117.52(\mathrm{C}-6 \mathrm{Ar}), 118.42$ (C-4a Ar), 123.14 (C-7 Ar), 124.64 (C-5 Ar), 125.77 (C-2', 6' Ar), 127.75 (C-2", 6" Ar), 128.30 (C-3", 5" Ar), 128.38 (C-4" Ar), 128.66 (C-8 Ar), 130.27 (C-4', Ar), 130.46 (C-3', 5' Ar), 132.88 (C-1' Ar), 138.30 (C-1'” Ar), $144.14(\mathrm{C}-8 \mathrm{a} \mathrm{Ar}), 151.92(\mathrm{C}=\mathrm{N}), 155.14(\underline{\mathrm{C}}=\mathrm{CH})$. Mass spectrum, $m / z\left(I_{\text {rel }}, \%\right): 327[\mathrm{M}]^{+}(36), 236(46)$, 206 (100). Found, $m / z: 328.1337[\mathrm{M}+\mathrm{H}]^{+}, \mathrm{C}_{22} \mathrm{H}_{18} \mathrm{NO}_{2}$. Calculated, $m / z: 328.1338$.

2-Phenyl-4H-chromen-4-one $\mathrm{O}$-(2-bromobenzyl)oxime (3c)

Yield 74\%; mp 82-83 ${ }^{\circ} \mathrm{C} ;{ }^{1} \mathrm{H}$ NMR (300 MHz, $\mathrm{CDCl}_{3}$ ) $\delta 5.37\left(\mathrm{~s}, 2 \mathrm{H}, \mathrm{OCH}_{2}\right), 7.16-7.36(\mathrm{~m}, 6 \mathrm{H}, \mathrm{C}=\mathrm{CH}, \mathrm{H}-6,7$ Ar, H-4", 5", 6" Ar), 7.45-7.54 (m, 4H, H-8, 3', 4', 5' Ar), 7.60 (d, 1H, $J 8.7 \mathrm{~Hz}, \mathrm{H}-3$ " Ar), 7.91-7.92 (m, 2H, $\mathrm{H}-2^{\prime}, 6^{\prime} \mathrm{Ar}$ ), 8.03 (dd, 1H, J 7.5, 0.3 Hz, H-5 Ar); ${ }^{13} \mathrm{C}$ NMR $\left(75 \mathrm{MHz}, \mathrm{CDCl}_{3}\right) \delta 75.39\left(\mathrm{OCH}_{2}\right), 94.00(\mathrm{C}=\mathrm{CH}), 117.50$ (C-6 Ar), 118.30 (C-4a Ar), 122.84 (C-Br), 123.24 (C-5 Ar), 124.66 (C-7 Ar), 125.79 (C-2', 6' Ar), 127.30 (C-4" 
Ar), 128.68 (C-5” Ar), 128.94 (C-3', 5' Ar), 129.65 (C-6" Ar), 130.32 (C-4' Ar), 130.53 (C-8 Ar), 132.55 (C-3” Ar), 132.89 (C-1'), 137.94 (C-1" Ar), 144.57 (C-8a Ar), 151.91 $(\mathrm{C}=\mathrm{N}), 155.38(\underline{\mathrm{C}}=\mathrm{CH})$. Mass spectrum, $m / z\left(I_{\mathrm{rel}}, \%\right): 405$ $\left[\mathrm{M}\left({ }^{79} \mathrm{Br}\right)\right]^{+}(12), 236(52), 206$ (100). Found, $m / z: 406.0446$ $[\mathrm{M}+\mathrm{H}]^{+}, \mathrm{C}_{22} \mathrm{H}_{17} \mathrm{BrNO}_{2}$. Calculated, $m / z: 406.0443$.

2-Phenyl-4H-chromen-4-one O-(2-bromoallyl)oxime (3d)

Yield $72 \%$; mp 79-80 ${ }^{\circ} \mathrm{C} ;{ }^{1} \mathrm{H} \mathrm{NMR}\left(300 \mathrm{MHz}, \mathrm{CDCl}_{3}\right)$ $\delta 4.83\left(\mathrm{~s}, 2 \mathrm{H}, \mathrm{OCH}_{2}\right), 5.67(\mathrm{~s}, 1 \mathrm{H}, \mathrm{BrC}=\mathrm{C} \underline{\mathrm{H}}), 5.97(\mathrm{~s}, 1 \mathrm{H}$, $\mathrm{BrC}=\mathrm{C} \underline{\mathrm{H}}), 7.16(\mathrm{~s}, 1 \mathrm{H}, \mathrm{C}=\mathrm{C} \underline{\mathrm{H}}), 7.22-7.32(\mathrm{~m}, 2 \mathrm{H}, \mathrm{H}-6$, 7 Ar), 7.45-7.49 (m, 4H, H-8, 3', 4', 5' Ar), 7.91-7.92 (m, 2H, H-2', 6' Ar), 8.04 (dd, 1H, J 7.8, $1.2 \mathrm{~Hz}, \mathrm{H}-5$ $\mathrm{Ar}) ;{ }^{13} \mathrm{C} \mathrm{NMR}\left(75 \mathrm{MHz}, \mathrm{CDCl}_{3}\right) \delta 77.45\left(\mathrm{OCH}_{2}\right), 93.79$ $(\mathrm{C}=\mathrm{CH}), 117.49\left(\mathrm{BrC}=\mathrm{CH}_{2}\right), 117.55(\mathrm{C}-6 \mathrm{Ar}), 118.05$ (C-4a Ar), 123.24 (C-5 Ar), 124.72 (C-7 Ar), 125.81 (C-2', 6' Ar), 128.71 (C-3', 5' Ar), $129.66\left(\mathrm{BrC}=\mathrm{CH}_{2}\right), 130.42$ (C-4' Ar), 130.68 (C-8 Ar), 132.79 (C-1' Ar), 144.92 (C-8a Ar), $151.96(\mathrm{C}=\mathrm{N}), 155.31(\underline{\mathrm{C}}=\mathrm{CH})$. Mass spectrum, $\mathrm{m} / \mathrm{z}$ $\left(I_{\text {rel }}, \%\right): 355\left[\mathrm{M}\left({ }^{79} \mathrm{Br}\right)\right]^{+}(13), 236(36), 206$ (100). Found, $\mathrm{m} / z: 356.0287[\mathrm{M}+\mathrm{H}]^{+}, \mathrm{C}_{18} \mathrm{H}_{15} \mathrm{BrNO}_{2}$. Calculated, $\mathrm{m} / z$ : 356.0286 .

\section{2-Phenyl-4H-chromen-4-one $O$-(3-methylbut-2-en-1-yl)} oxime (3e)

Yield 64\%; ${ }^{1} \mathrm{H}$ NMR $\left(300 \mathrm{MHz}, \mathrm{CDCl}_{3}\right) \delta 1.82(\mathrm{~s}, 3 \mathrm{H}$, $\left.\mathrm{C}_{3}\right), 1.84\left(\mathrm{~s}, 3 \mathrm{H}, \mathrm{C}_{3}\right), 4.75\left(\mathrm{~d}, 2 \mathrm{H}, J 7.0 \mathrm{~Hz}, \mathrm{OC}_{2}\right)$, $5.51\left(\mathrm{t}, 1 \mathrm{H}, J 7.0 \mathrm{~Hz}, \underline{\mathrm{HC}}=\mathrm{C}\left(\mathrm{CH}_{3}\right)_{2}\right), 7.10(\mathrm{~s}, 1 \mathrm{H}, \mathrm{C}=\mathrm{C} \underline{\mathrm{H}})$, 7.20-7.26 (td, $1 \mathrm{H}, J$ 7.2, $1.3 \mathrm{~Hz}, \mathrm{H}-6 \mathrm{Ar}), 7.28-7.32$ (dd, $1 \mathrm{H}, J$ 8.0, $1.3 \mathrm{~Hz}, \mathrm{H}-8 \mathrm{Ar}$ ), 7.40-7.54 (m, 4H, H-7, H-3', 4', 5' Ar), 7.86-7.96 (m, 2H, H-2', 6' Ar), 8.06 (dd, 1H, J 7.95, $1.3 \mathrm{~Hz}, \mathrm{H}-5 \mathrm{Ar}) ;{ }^{13} \mathrm{C}$ NMR (75 MHz, $\left.\mathrm{CDCl}_{3}\right) \delta 18.28$ $\left(\underline{\mathrm{C}}_{3}\right), 25.94\left(\underline{\mathrm{CH}}_{3}\right), 70.91\left(\mathrm{OCH}_{2}\right), 94.14(\mathrm{C}=\underline{\mathrm{CH}}), 117.48$ $(\mathrm{C}-8 \mathrm{Ar}), 118.64(\mathrm{C}-4 \mathrm{a} \mathrm{Ar}), 120.60\left(\mathrm{HC}=\mathrm{C}\left(\mathrm{CH}_{3}\right)_{2}\right), 123.06$ (C-5 Ar), 124.57 (C-6 Ar), 125.72 (C-2', 6' Ar), 128.60 (C-3', 5' Ar), 130.16 (C-4' Ar), 130.30 (C-7 Ar), 132.97 (C-1' $\mathrm{Ar}), 137.57\left(\mathrm{HC}=\mathrm{C}\left(\mathrm{CH}_{3}\right)_{2}\right), 143.63(\mathrm{C}-8 \mathrm{a} \mathrm{Ar}), 151.91$ $(\mathrm{C}=\mathrm{N}), 154.92(\underline{\mathrm{C}}=\mathrm{CH})$. Found, $m / z: 306.1494[\mathrm{M}+\mathrm{H}]^{+}$, $\mathrm{C}_{20} \mathrm{H}_{20} \mathrm{NO}_{2}$. Calculated, $m / z: 306.1494$.

\section{2-Phenyl-4H-chromen-4-one O-cinnamyloxime (3f)}

Yield 60\%; mp 68-69 ${ }^{\circ} \mathrm{C} ;{ }^{1} \mathrm{H}$ NMR $\left(300 \mathrm{MHz}, \mathrm{CDCl}_{3}\right)$ $\delta 4.93\left(\mathrm{~d}, 2 \mathrm{H}, J 6.2 \mathrm{~Hz}, \mathrm{OC} \underline{\mathrm{H}}_{2}\right), 6.49-6.62(\mathrm{dt}, 1 \mathrm{H}, J$ 16.0, $6.2 \mathrm{~Hz}, \underline{\mathrm{HC}}=\mathrm{CHPh}), 6.76(\mathrm{~d}, 1 \mathrm{H}, J 16.0 \mathrm{~Hz}, \mathrm{HC}=\mathrm{C} \underline{\mathrm{HPh}})$, 7.16 (s, $1 \mathrm{H}, \mathrm{C}=\mathrm{C} \mathrm{H}), 7.22-7.56$ (m, 11H, H-6, 7, 8, 3', 4', 5', 2", 3", 4", 5", 6" Ar), 7.87-7.97 (m, 2H, H-2', 6' Ar), 8.09 (dd, $1 \mathrm{H}, J$ 7.9, $1.2 \mathrm{~Hz}, \mathrm{H}-5 \mathrm{Ar}) ;{ }^{13} \mathrm{C}$ NMR $(75 \mathrm{MHz}$, $\left.\mathrm{CDCl}_{3}\right) \delta 74.97\left(\mathrm{OCH}_{2}\right), 94.00(\mathrm{C}=\underline{\mathrm{C}} \mathrm{H}), 117.55(\mathrm{C}-8 \mathrm{Ar})$, 118.44 (C-4a Ar), 123.14 (C-5 Ar), 124.68 (C-6 Ar), 125.76 (C-2', 6' Ar), 125.94 (HC=CHPh), 126.65 (C-2", 6” Ar), 127.76 (C-4” Ar), 128.59 (C-3', 5' Ar), 128.68 (C-3”, 5”
Ar), 130.30 (C-4' Ar), 130.48 (C-7 Ar), 132.86 (C-1' Ar), $133.03(\mathrm{HC}=\underline{\mathrm{CHPh}}), 136.83(\mathrm{C}-1$ " Ar), 144.04 (C-8a Ar), $151.92(\mathrm{C}=\mathrm{N}), 155.14(\mathrm{C}=\mathrm{CH})$. Found, $m / z: 354.1496$ $[\mathrm{M}+\mathrm{H}]^{+}, \mathrm{C}_{20} \mathrm{H}_{20} \mathrm{NO}_{2}$. Calculated, $m / z: 354.1494$.

6-Hydroxy-2-phenyl-4H-chromen-4-one O-methyloxime (3g)

Yield 54\%; mp 149-150 ${ }^{\circ} \mathrm{C}$; ${ }^{1} \mathrm{H}$ NMR $(300 \mathrm{MHz}$, $\left.\mathrm{CDCl}_{3}\right) \delta 4.30\left(\mathrm{~s}, 3 \mathrm{H}, \mathrm{OCH}_{3}\right), 6.93(\mathrm{dd}, 1 \mathrm{H}, J 9.0,2.9 \mathrm{~Hz}$, H-7 Ar), 6.96 (s, 1H, C=CH), $7.19(\mathrm{~d}, 1 \mathrm{H}, J 9.0 \mathrm{~Hz}, \mathrm{H}-8$ $\mathrm{Ar}$ ), 7.31 (d, 1H, J $2.9 \mathrm{~Hz}, \mathrm{H}-5 \mathrm{Ar}), 7.40-7.50$ (m, 3H, H-3', 4', 5' Ar), 7.70-7.80 (m, 2H, H-2', 6' Ar); ${ }^{13} \mathrm{C}$ NMR $\left(75 \mathrm{MHz}, \mathrm{CDCl}_{3}\right) \delta 60.66\left(\mathrm{OCH}_{3}\right), 92.00(\mathrm{C}=\underline{\mathrm{CH}}), 106.50$ (C-5 Ar), 118.30 (C-7 Ar), 118.40 (C-4a Ar), 118.69 (C-8 Ar), 125.18 (C-2', 6' Ar), 128.39 (C-3', 5' Ar), 129.92 (C-4' Ar), 132.78 (C-1' Ar), 143.92 (C-8a Ar), 145.54 (C-6 $\operatorname{Ar}), 154.23(\mathrm{C}=\mathrm{N}), 155.17(\mathrm{C}=\mathrm{CH})$. Found, $m / z: 268.0975$ $[\mathrm{M}+\mathrm{H}]^{+}, \mathrm{C}_{16} \mathrm{H}_{14} \mathrm{NO}_{3}$. Calculated, $\mathrm{m} / \mathrm{z}: 268.0974$.

\section{6-Hydroxy-2-phenyl-4H-chromen-4-one O-benzyloxime} (3h)

Yield 58\%; mp 150-151 ${ }^{\circ} \mathrm{C}$; ${ }^{1} \mathrm{H}$ NMR (300 MHz, $\left.\mathrm{CDCl}_{3}\right) \delta 5.20\left(\mathrm{~s}, 2 \mathrm{H}, \mathrm{OCH}_{2}\right), 6.95(\mathrm{dd}, 1 \mathrm{H}, J 9.0,3.0 \mathrm{~Hz}$, H-7 Ar), 7.05 (s, 1H, C=CH), $7.18(\mathrm{~d}, 1 \mathrm{H}, J 9.0 \mathrm{~Hz}$, H-8 Ar), 7.28-7.35 (m, 6H, H-5, 2", 3", 4", 5", 6" Ar), 7.37-7.45 (m, 3H, H-3', 4', 5' Ar), 7.81-7.84 (m, 2H, H-2', 6 ' Ar); ${ }^{13} \mathrm{C} \mathrm{NMR}\left(75 \mathrm{MHz}, \mathrm{CDCl}_{3}\right) \delta 75.84\left(\mathrm{OCH}_{2}\right), 92.70$ $(\mathrm{C}=\underline{\mathrm{C}} \mathrm{H}), 106.96(\mathrm{C}-5 \mathrm{Ar}), 118.37$ (C-4a Ar), $118.62(\mathrm{C}-7$ Ar), 119.08 (C-8 Ar), 125.67 (C-2', 6' Ar), 127.66 (C-2”, 6" Ar), 128.08 (C-3", 5" Ar), 128.24 (C-4" Ar), 128.53 (C-3', 5' Ar), 130.16 (C-4', Ar), 132.85 (C-1' Ar), 138.02 (C-1" Ar), 145.12 (C-8a Ar), 145.85 (C-6 Ar), 153.86 $(\mathrm{C}=\mathrm{N}), 156.54(\underline{\mathrm{C}}=\mathrm{CH})$. Found, $m / z: 344.1287[\mathrm{M}+\mathrm{H}]^{+}$, $\mathrm{C}_{22} \mathrm{H}_{18} \mathrm{NO}_{3}$. Calculated, $m / z$ : 344.1287 .

6-Hydroxy-2-phenyl-4H-chromen-4-one O-(2-bromobenzyl) oxime (3i)

Yield 76\%; mp 123-124 ${ }^{\circ} \mathrm{C}$; ${ }^{1} \mathrm{H}$ NMR (300 MHz, $\left.\mathrm{CDCl}_{3}\right) \delta 5.28\left(\mathrm{~s}, 2 \mathrm{H}, \mathrm{OCH}_{2}\right), 6.95(\mathrm{dd}, 1 \mathrm{H}, J 8.9,3.0 \mathrm{~Hz}$, H-7 Ar), 7.11 (s, 1H, C=CH), 7.15-7.26 (m, 2H, H-8, 4", Ar), 7.28-7.39 (m, 2H, H-5, 5" Ar), 7.41-7.48 (m, 3H, H-3', 4', 5' Ar), 7.51 (d, 1H, J 7.6 Hz, H-6" Ar), 7.58 (d, 1H, J 7.6 Hz, H-3" Ar), 7.81-7.91 (m, 2H, H-2', 6' $\mathrm{Ar}) ;{ }^{13} \mathrm{C}$ NMR $\left(75 \mathrm{MHz}, \mathrm{CDCl}_{3}\right) \delta 74.89\left(\mathrm{OCH}_{2}\right), 92.29$ $(\mathrm{C}=\mathrm{CH}), 106.73$ (C-5 Ar), 118.32 (C-4a Ar), 118.39 (C-7 Ar), 118.93 (C-8 Ar), 122.52 (C-Br), 125.35 (C-2', 6' Ar), 127.16 (C-5”), 128.45 (C-3', 4', 5' Ar), 128.91 (C-4"' Ar), 129.66 (C-3" Ar), 132.28 (C-6" Ar), 132.78 (C-1' Ar), 137.64 (C-1" Ar), 145.00 (C-8a Ar), 145.66 (C-6 $\mathrm{Ar}), 154.54(\mathrm{C}=\mathrm{N}), 155.50(\mathrm{C}=\mathrm{CH})$. Found, $m / z: 422.0398$ $[\mathrm{M}+\mathrm{H}]^{+}, \mathrm{C}_{22} \mathrm{H}_{17} \mathrm{BrNO}_{3}$. Calculated, $\mathrm{m} / \mathrm{z}: 422.0392$. 
6-Hydroxy-2-phenyl-4H-chromen-4-one O-(2-bromoallyl) oxime (3j)

Yield 69\%; mp 129-130 ${ }^{\circ} \mathrm{C} ;{ }^{1} \mathrm{H}$ NMR (300 MHz, $\left.\mathrm{CDCl}_{3}\right) \delta 4.76\left(\mathrm{~s}, 2 \mathrm{H}, \mathrm{OCH}_{2}\right), 5.61(\mathrm{~s}, 1 \mathrm{H}, \mathrm{BrC}=\mathrm{C} \underline{\mathrm{H}}), 5.93$ $(\mathrm{s}, 1 \mathrm{H}, \mathrm{BrC}=\mathrm{CH}), 6.96(\mathrm{dd}, 1 \mathrm{H}, J$ 9.0, 3.0 Hz, H-7 Ar), $7.07(\mathrm{~s}, 1 \mathrm{H}, \mathrm{C}=\mathrm{CH}), 7.21(\mathrm{~d}, 1 \mathrm{H}, J 9.0 \mathrm{~Hz}, \mathrm{H}-8 \mathrm{Ar}), 7.32$ (d, 1H, J 3.0 Hz, H-5 Ar), 7.40-7.50 (m, 3H, H-3', 4', 5', Ar), 7.80-7.90 (m, 2H, H-2', 6' Ar); ${ }^{13} \mathrm{C}$ NMR (75 MHz, $\left.\mathrm{CDCl}_{3}\right) \delta 77.14\left(\mathrm{OCH}_{2}\right), 92.53(\mathrm{C}=\underline{\mathrm{CH}}), 106.99(\mathrm{C}-5 \mathrm{Ar})$, $117.37\left(\mathrm{BrC}=\underline{\mathrm{CH}}_{2}\right), 118.14(\mathrm{C}-4 \mathrm{a} \mathrm{Ar}), 118.64(\mathrm{C}-8 \mathrm{Ar})$, 119.30 (C-7 Ar), 125.62 (C-2', 6’ Ar), 128.57 (C-3', 5’ Ar), $129.42\left(\mathrm{Br} \underline{\mathrm{C}}=\mathrm{CH}_{2}\right), 130.27$ (C-4', Ar), 132.78 (C-1' Ar), 145.76 (C-6 Ar), 145.88 (C-8a Ar), 153.93 (C=N), 155.80 $(\underline{\mathrm{C}}=\mathrm{CH})$. Found, $m / z$ : $372.0235[\mathrm{M}+\mathrm{H}]^{+}, \mathrm{C}_{18} \mathrm{H}_{15} \mathrm{BrNO}_{3}$. Calculated, $m / z: 372.0235$.

6-Hydroxy-2-phenyl-4H-chromen-4-one $\mathrm{O}$-(3-methylbut2-en-1-yl)oxime (3k)

Yield 67\%; mp 82-84 ${ }^{\circ} \mathrm{C} ;{ }^{1} \mathrm{H}$ NMR $\left(300 \mathrm{MHz}, \mathrm{CDCl}_{3}\right.$ ) $\delta 1.77\left(\mathrm{~s}, 3 \mathrm{H}, \mathrm{CH}_{3}\right), 1.80\left(\mathrm{~s}, 3 \mathrm{H}, \mathrm{CH}_{3}\right), 4.74(\mathrm{~d}, 2 \mathrm{H}$, $\left.J 7.0 \mathrm{~Hz}, \mathrm{OCH}_{2}\right), 5.56\left(\mathrm{t}, 1 \mathrm{H}, J 7.0 \mathrm{~Hz}, \underline{\mathrm{HC}}=\mathrm{C}\left(\mathrm{CH}_{3}\right)_{2}\right), 7.00$ (dd, 1H, J 8.9, $2.8 \mathrm{~Hz}, \mathrm{H}-7 \mathrm{Ar}), 7.06$ (s, 1H, C=C $\underline{\mathrm{H}}$ ), 7.19 (d, 1H, J 8.9 Hz, H-8 Ar), 7.43-7.51 (m, 4H, H-5, 3', 4', 5' Ar), 7.80-7.92 (m, 2H, H-2', 6' Ar); ${ }^{13} \mathrm{C}$ NMR (75 MHz, $\left.\mathrm{CDCl}_{3}\right) \delta 18.30\left(\underline{\mathrm{CH}}_{3}\right), 25.98\left(\underline{\mathrm{CH}}_{3}\right), 70.71\left(\mathrm{OCH}_{2}\right), 93.24$ $(\mathrm{C}=\underline{\mathrm{CH}}), 107.54$ (C-5 Ar), 118.67 (C-4a Ar), 118.96 (C-7 Ar), $119.22(\mathrm{C}-8 \mathrm{Ar}), 120.26\left(\mathrm{HC}=\mathrm{C}\left(\mathrm{CH}_{3}\right)_{2}\right), 125.36$ (C-2', 6' Ar), 128.61 (C-3', 5' Ar), 130.22 (C-4' Ar), $132.89(\mathrm{C}-1$ ' Ar $), 138.08\left(\mathrm{HC}=\underline{\mathrm{C}}\left(\mathrm{CH}_{3}\right)_{2}\right), 144.74(\mathrm{C}-8 \mathrm{a}$ Ar), $146.30(\mathrm{C}-6$ Ar), $152.87(\mathrm{C}=\mathrm{N}), 155.35(\mathrm{C}=\mathrm{CH})$. Found, $m / z: 322.1445[\mathrm{M}+\mathrm{H}]^{+}, \mathrm{C}_{20} \mathrm{H}_{20} \mathrm{NO}_{3}$. Calculated, $m / z: 322.1443$.

\section{6-Hydroxy-2-phenyl-4H-chromen-4-one O-cinnamyloxime} (3I)

Yield 87\%; mp 83-84 ${ }^{\circ} \mathrm{C} ;{ }^{1} \mathrm{H}$ NMR $\left(300 \mathrm{MHz}, \mathrm{CDCl}_{3}\right)$ $\delta 4.88\left(\mathrm{~d}, 2 \mathrm{H}, J 6.0 \mathrm{~Hz}, \mathrm{OCH}_{2}\right), 6.44-6.56(\mathrm{dt}, 1 \mathrm{H}, J$ 16.0, $6.0 \mathrm{~Hz}, \underline{\mathrm{HC}}=\mathrm{CHPh}), 6.71(\mathrm{~d}, 1 \mathrm{H}, J 16.0 \mathrm{~Hz}, \mathrm{HC}=\mathrm{C} \underline{\mathrm{HPh}})$, 6.99 (dd, 1H, J 8.9, $2.9 \mathrm{~Hz}, \mathrm{C}-7), 7.02$ (d, 1H, J 8.9 Hz, C-8), 7.09 (s, 1H, C=C $\underline{\mathrm{H}})$, 7.24-7.55 (m, 9H, H-5, 3', 4', 5', 2", 3", 4", 5', 6" Ar), 7.84-7.92 (m, 2H, H-2', 6' $\mathrm{Ar}) ;{ }^{13} \mathrm{C} \mathrm{NMR}\left(75 \mathrm{MHz}, \mathrm{CDCl}_{3}\right) \delta 74.83\left(\mathrm{OCH}_{2}\right), 93.12$ $(\mathrm{C}=\underline{\mathrm{CH}}$ ), 107.55 (C-5 Ar), 118.74 (C-4a Ar), 118.92 (C-7 $\mathrm{Ar}), 119.11$ (C-8 Ar), 125.76 (HC=CHPh, C-2', 6' Ar), 126.62 (C-2", 6" Ar), 127.74 (C-4"'Ar), 128.56 (C-3', 5' Ar), 128.64 (C-3', 5” Ar), 130.24 (C-4' Ar), 132.91 (C-1' Ar), 133.10 (HC=CHPh), 136.75 (C-1" 'Ar), 144.57 (C-8a Ar), 146.53 (C-6 Ar), $152.94(\mathrm{C}=\mathrm{N}), 155.6(\underline{\mathrm{C}}=\mathrm{CH})$. Found, $m / z: 370.1444[\mathrm{M}+\mathrm{H}]^{+}, \mathrm{C}_{24} \mathrm{H}_{20} \mathrm{NO}_{3}$. Calculated, $m / z: 370.1443$.
Synthesis of 2-alkyloxyisoindoline-1,3-diones

A solution of $N$-hydroxyphthalimide $(1 \mathrm{mmol})$, an alkyl bromide $(0.5 \mathrm{mmol})$, and $\mathrm{K}_{2} \mathrm{CO}_{3}(2 \mathrm{mmol})$ in DMSO $(1 \mathrm{~mL})$, was stirred at room temparature for $2-3 \mathrm{~h}$. The reaction mixture was treated whit distilled water $(10 \mathrm{~mL})$, filtered, washed whith water and dried to provide products 3c-f.

2-((2-Bromobenzyl)oxy)isoindoline-1,3-dione (4c)

Yield 92\%; mp 159-160 ${ }^{\circ} \mathrm{C}$; ${ }^{1} \mathrm{H}$ NMR (300 MHz, $\left.\mathrm{CDCl}_{3}\right) \delta 5.37\left(\mathrm{~s}, 2 \mathrm{H}, \mathrm{OC}_{2}\right), 7.25(\mathrm{td}, 1 \mathrm{H}, J 7.5,1.3 \mathrm{~Hz}$, H-4' Ar), 7.37 (t, 1H, J 7.5 Hz, H-5' Ar), 7.56 (d, 1H, J 7.5 Hz, H-3' Ar), 7.66 (dd, 1H, J 7.5, 1.3 Hz, H-6' Ar), 7.72-7.79 (m, 2H, H-4, 7 Ar), 7.79-7.86 (m, 2H, H-5, 6 $\mathrm{Ar}) ;{ }^{13} \mathrm{C}$ NMR $\left(75 \mathrm{MHz}, \mathrm{CDCl}_{3}\right) \delta 78.67\left(\mathrm{OCH}_{2}\right), 123.55$ (C-5, 6 Ar), 124.55 (C-Br), 127.65 (C-5' Ar), 128.33 (C-3a, 1a Ar), 130.72 (C-4' Ar), 131.75 (C-6' Ar), 132.89 (C-3’ Ar), 133.66 (C-1' Ar), 134.47 (C-4, 7 Ar), 163.38 $(\mathrm{C}=\mathrm{O})$. Mass spectrum, $m / z\left(I_{\text {rel }}, \%\right): 331\left[\mathrm{M}\left({ }^{79} \mathrm{Br}\right)\right]^{+}, 169$ (100), 76 (22).

\section{2-((2-Bromoallyl)oxy)isoindoline-1,3-dione (4d)}

Yield 97\%; mp 110-111 ${ }^{\circ} \mathrm{C}$; ${ }^{1} \mathrm{H}$ NMR (300 MHz, $\left.\mathrm{CDCl}_{3}\right) \delta 4.85\left(\mathrm{~s}, 2 \mathrm{H}, \mathrm{OC}_{2}\right), 5.79(\mathrm{~s}, 1 \mathrm{H}, \mathrm{BrC}=\mathrm{C} \underline{\mathrm{H}}), 6.16$ (s, $1 \mathrm{H}, \mathrm{BrC}=\mathrm{C} \underline{\mathrm{H}}), 7.75-7.80(\mathrm{~m}, 2 \mathrm{H}, \mathrm{H}-4,7 \mathrm{Ar}), 7.85-7.89$ (m, 2H, H-5, $6 \mathrm{Ar}) ;{ }^{13} \mathrm{C} \mathrm{NMR}\left(75 \mathrm{MHz}, \mathrm{CDCl}_{3}\right) \delta 81.08$ $\left(\mathrm{OCH}_{2}\right), 122.82\left(\mathrm{BrC}=\underline{\mathrm{CH}}_{2}\right), 123.71(\mathrm{C}-5,6 \mathrm{Ar}), 124.66$ $(\mathrm{BrC}=\mathrm{CH}), 128.70(\mathrm{C}-3 \mathrm{a}, 1 \mathrm{a} \mathrm{Ar}), 134.70(\mathrm{C}-4,7 \mathrm{Ar})$, $163.28(\mathrm{C}=\mathrm{O})$. Mass spectrum, $m / z\left(I_{\text {rel }}, \%\right): 281\left[\mathrm{M}\left({ }^{79} \mathrm{Br}\right)\right]^{+}$, 202 (100), 162 (18). Found, $m / z: 281.9761[\mathrm{M}+\mathrm{H}]^{+}$, $\mathrm{C}_{11} \mathrm{H}_{9} \mathrm{BrNO}_{3}$. Calculated, $m / z: 281.9766$.

2-((3-Methylbut-2-en-1-yl)oxy)isoindoline-1,3-dione (4e)

Yield 78\%; mp 95-96 ${ }^{\circ} \mathrm{C} ;{ }^{1} \mathrm{H}$ NMR (300 MHz, $\left.\mathrm{CDCl}_{3}\right) \delta$ 1.75 (s, 3H, $\left.\mathrm{CH}_{3}\right), 1.79$ (s, 3H, $\left.\mathrm{CH}_{3}\right), 4.74$ (d, 2H, J 7.6 Hz, $\left.\mathrm{OCH}_{2}\right), 5.55(\mathrm{t}, 1 \mathrm{H}, J 7.6 \mathrm{~Hz}, \underline{\mathrm{HC}}=\mathrm{C}), 7.71-7.81(\mathrm{~m}$, $2 \mathrm{H}, \mathrm{H}-4,7 \mathrm{Ar}), 7.82-7.89$ (m, 2H, H-5, $6 \mathrm{Ar}) ;{ }^{13} \mathrm{C} \mathrm{NMR}$ $\left(75 \mathrm{MHz}, \mathrm{CDCl}_{3}\right) \delta 18.13\left(\underline{\mathrm{CH}}_{3}\right), 25.97\left(\underline{\mathrm{CH}}_{3}\right), 74.06$ $\left(\mathrm{OCH}_{2}\right), 117.04\left(\mathrm{HC}=\underline{\mathrm{C}}\left(\mathrm{CH}_{3}\right)_{2}\right), 123.45(\mathrm{C}-5,6 \mathrm{Ar}), 128.92$ (C-3a, 1a Ar), 134.41 (C-4, $7 \mathrm{Ar}), 143.72\left(\mathrm{HC}=\underline{\mathrm{C}}\left(\mathrm{CH}_{3}\right)_{2}\right)$, $163.86(\mathrm{C}=\mathrm{O})$. Found, $m / z: 232.0971[\mathrm{M}+\mathrm{H}]^{+}, \mathrm{C}_{13} \mathrm{H}_{14} \mathrm{NO}_{3}$. Calculated, $m / z: 232.0974$.

\section{2-(Cinnamyloxy)isoindoline-1,3-dione (4f)}

Yield 87\%; mp 144-145 ${ }^{\circ} \mathrm{C}$; ${ }^{1} \mathrm{H}$ NMR (300 MHz, $\left.\mathrm{CDCl}_{3}\right) \delta 4.88\left(\mathrm{~d}, 2 \mathrm{H}, J 7.0 \mathrm{~Hz}, \mathrm{OCH}_{2}\right), 6.48$ (dt, 1H, J 15.9, $7.0 \mathrm{~Hz}, \underline{\mathrm{HC}}=\mathrm{CHPh}), 6.69(\mathrm{~d}, 1 \mathrm{H}, J 15.9 \mathrm{~Hz}, \mathrm{HC}=\mathrm{C} \underline{\mathrm{HPh}})$, 7.22-7.43 (m, 5H, H-2', 3', 4', 5', 6' Ar), 7.71-7.78 (m, 2H, H-4, 7 Ar), 7.78-7.86 (m, 2H, H-5, 6 Ar); ${ }^{13} \mathrm{C} \mathrm{NMR}$ $\left(75 \mathrm{MHz}, \mathrm{CDCl}_{3}\right) \delta 78.67\left(\mathrm{OCH}_{2}\right), 122.01(\mathrm{HC}=\mathrm{CHPh})$, 
123.56 (C-5, 6 Ar), 126.93 (C-4' Ar), 128.47 (C-2', 6' Ar), 128.64 (C-3', 5' Ar), 128.80 (C-3a, 1a Ar), 134.50 (C-4, 7 Ar), 135.77 (C-1' Ar), 137.53 (HC= $\underline{\mathrm{C} H P h}), 163.86(\mathrm{C}=\mathrm{O})$. Found, $m / z: 302.0794\left[\mathrm{M}+\mathrm{Na}^{+}, \mathrm{C}_{17} \mathrm{H}_{13} \mathrm{NaNO}_{3}\right.$. Calculated, $\mathrm{m} / \mathrm{z}: 302.0793$.

\section{Synthesis of $O$-alkylhydroxylamine hydrochlorides}

To a stirred solution of hydrazine sulfate $(1.2 \mathrm{mmol})$, $\mathrm{K}_{2} \mathrm{CO}_{3}(1.2 \mathrm{mmol})$ and water $(2.0 \mathrm{~mL})$ in THF $(2.0 \mathrm{~mL})$, was added the corresponding $O$-alkyl- $N$-hydroxyphthalimide $(1.0 \mathrm{mmol})$, and the mixture was refluxed during $5 \mathrm{~h}$, then distilled water $(10 \mathrm{~mL})$ was added. The resulting mixture was extracted with ethyl ether $(3 \times 10 \mathrm{~mL})$, the organic phase was dried with sodium sulfate, filtrated and the solvent evaporated under reduced pressure. The residue was chromatographed by CC (column chromatography) using silica gel, Hex:AcOEt 7:3 to give the corresponding hydroxylamine. Subsequently the hydroxilamine was disolved in dried ethyl ether and $\mathrm{HCl}(\mathrm{g})$ was bubbled to obtain the salt (3c-f).

\section{O-(2-Bromobenzyl)hydroxylamine hydrochloride (2c)}

Yield 78\%; mp 98-99 ${ }^{\circ} \mathrm{C} ;{ }^{1} \mathrm{H}$ NMR $\left(300 \mathrm{MHz}, \mathrm{CDCl}_{3}\right)$ $\delta 4.76\left(\mathrm{~s}, 2 \mathrm{H}, \mathrm{OCH}_{2}\right), 7.18(\mathrm{td}, 1 \mathrm{H}, J$ 7.5, $1.5 \mathrm{~Hz}, \mathrm{H}-5 \mathrm{Ar})$, 7.33 (t, $1 \mathrm{H}, J 7.5 \mathrm{~Hz}, \mathrm{H}-4 \mathrm{Ar}), 7.44$ (dd, $1 \mathrm{H}, J 7.5,1.5 \mathrm{~Hz}$, H-6 Ar), 7.57 (d, $1 \mathrm{H}, J 7.5 \mathrm{~Hz}, \mathrm{H}-3 \mathrm{Ar}) ;{ }^{13} \mathrm{C}$ NMR $(75 \mathrm{MHz}$, $\left.\mathrm{CDCl}_{3}\right) \delta 77.14\left(\mathrm{OCH}_{2}\right), 123.54(\mathrm{C}-\mathrm{Br}), 127.35(\mathrm{C}-5 \mathrm{Ar})$, 129.31 (C-4 Ar), 129.95 (C-6 Ar), 132.74 (C-3 Ar), 136.90 $\left(\mathrm{CCH}_{2} \mathrm{O}\right.$ Ar). Mass spectrum, $m / z\left(I_{\text {rel }}, \%\right): 201\left[\mathrm{M}\left({ }^{(9} \mathrm{Br}\right)\right]^{+}$, 169 (100), 90 (25).

\section{O-(2-Bromoallyl)hydroxylaminehydrochloride (2d)}

Yield 87\%; mp 153- $154{ }^{\circ} \mathrm{C} ;{ }^{1} \mathrm{H} \mathrm{NMR}\left(300 \mathrm{MHz}, \mathrm{CDCl}_{3}\right)$ $\delta 4.30\left(\mathrm{~s}, 2 \mathrm{H}, \mathrm{OCH}_{2}\right), 5.70(\mathrm{~d}, 1 \mathrm{H}, J 1.2 \mathrm{~Hz}, \mathrm{BrC}=\mathrm{CH}), 5.93$ $(\mathrm{d}, 1 \mathrm{H}, J 1.2 \mathrm{~Hz}, \mathrm{BrC}=\mathrm{CH}) ;{ }^{13} \mathrm{C} \mathrm{NMR}\left(75 \mathrm{MHz}, \mathrm{CDCl}_{3}\right) \delta$ $79.60\left(\mathrm{OCH}_{2}\right), 119.05(\mathrm{BrC}=\mathrm{CH}), 129.71(\mathrm{BrC}=\mathrm{CH})$. Mass spectrum, $m / z\left(I_{\text {rel }}, \%\right): 151\left[\mathrm{M}\left({ }^{79} \mathrm{Br}\right)\right]^{+}, 119(61), 39(100)$.

O-(3-Methylbut-2-en-1-yl)hydroxylamine hydrochloride (2e)

Yield 76\%; mp 160-161 ${ }^{\circ} \mathrm{C}$; ${ }^{1} \mathrm{H}$ NMR $(300 \mathrm{MHz}$, acetone- $\left.d_{6}\right) \delta 1.72\left(\mathrm{~s}, 3 \mathrm{H}, \mathrm{CH}_{3}\right), 1.74\left(\mathrm{~s}, 3 \mathrm{H}, \mathrm{CH}_{3}\right), 4.92(\mathrm{~d}$, $\left.2 \mathrm{H}, J 7.4 \mathrm{~Hz}, \mathrm{OCH}_{2}\right), 5.37\left(\mathrm{t}, J 7.4 \mathrm{~Hz}, 1 \mathrm{H}, \underline{\mathrm{HC}}=\mathrm{C}\left(\mathrm{CH}_{3}\right)_{2}\right)$; ${ }^{13} \mathrm{C}$ NMR $\left(75 \mathrm{MHz}\right.$, acetone- $\left.d_{6}\right) \delta 18.20\left(\mathrm{CH}_{3}\right), 25.72$ $\left(\mathrm{CH}_{3}\right), 72.12\left(\mathrm{OCH}_{2}\right), 116.83\left(\mathrm{HC}=\mathrm{C}\left(\mathrm{CH}_{3}\right)_{2}\right), 143.12$ $\left(\mathrm{HC}=\underline{\mathrm{C}}\left(\mathrm{CH}_{3}\right)_{2}\right)$.

O-(3-Phenyl-2-propenyl)-1-hydroxylamine hydrochloride (2f)

Yield 90\%; mp 176-177 ${ }^{\circ} \mathrm{C}$; ${ }^{1} \mathrm{H}$ NMR $(300 \mathrm{MHz}$, $\left.\mathrm{CDCl}_{3}-\mathrm{CD}_{3} \mathrm{OD}\right) \delta 4.72\left(\mathrm{~d}, 2 \mathrm{H}, J 6.9 \mathrm{~Hz}, \mathrm{CH}_{2}\right), 6.23-6.34$ (dt, $1 \mathrm{H}, J 15.6,6.9 \mathrm{~Hz}, \underline{\mathrm{HC}}=\mathrm{CHPh}), 6.83(\mathrm{~d}, 1 \mathrm{H}, J 15.6 \mathrm{~Hz}$, $\mathrm{HC}=\mathrm{C} \underline{\mathrm{HPh}}$ ), 7.25-7.35 (m, 3H, H Ar), 7.40-7.45 (m, 2H, H $\mathrm{Ar}),{ }^{13} \mathrm{C} \mathrm{NMR}\left(75 \mathrm{MHz}, \mathrm{CDCl}_{3}-\mathrm{CD}_{3} \mathrm{OD}\right) \delta 75.74\left(\mathrm{OCH}_{2}\right)$, $119.76(\mathrm{H} \underline{\mathrm{C}}=\mathrm{CHPh}), 126.85(\mathrm{Ar}-2,6), 128.61(\mathrm{Ar}-3,5)$, $128.72(\mathrm{Ar}-5), 135.38(\mathrm{Ar}-1), 138.53(\mathrm{HC}=\underline{\mathrm{CHPh}})$.

\section{Antiproliferative activity}

\section{Cell lines and culture conditions}

Cells were obtained from American Type Cell Culture (ATCC, Bethesda, BD). Tumor derived cells that originated in multiple tissue sites were maintained as follow: PC-3 (prostate adenocarcinoma), MRC-5 (fibroblasts derived from lung tissue) and A-549 (lung: NSCLC alveolar basal epithelial-squamous) in Dulbecco's modified Eagle's medium with high glucose (Lonza), supplemented with $10 \%$ (v/v) fetal bovine serum (Biowest), $2 \mathrm{mM} \mathrm{L}$-glutamine, $5000 \mathrm{UI} \mathrm{mL}^{-1}$ penicillin and $5 \mathrm{mg} \mathrm{mL}^{-1}$ streptomycin.

MDA-MB-231 (breast adenocarcinoma) was grown in RPMI 1640 medium (Lonza) supplemented with $10 \%$ serum (Biowest) and $2 \mathrm{mM} \mathrm{L-glutamine,} 5000 \mathrm{UI} \mathrm{\textrm {mL } ^ { - 1 }}$ penicillin and $5 \mathrm{mg} \mathrm{mL}^{-1}$ streptomycin. Eight thousand cells were seeded in 96 well plates and grown in a 5\% $\mathrm{CO}_{2}$ atmosphere at $37^{\circ} \mathrm{C}$ for $24 \mathrm{~h}$ before treatment. Cells were screened for mycoplasma contamination before each experiment, by means of DAPI staining (Invitrogen) and fluorescence microscopy camera (Motic CamPro 282A).

\section{MTT assay}

Cancer cells from breast (MDA-MB-231), prostate (PC-3) and lung (A-549) and healthy fibroblasts from lung (MRC-5) were seeded in a 96-well plate (5000 cells well ${ }^{-1}$ ) for $24 \mathrm{~h}$. Cells were then treated with concentrations in a range of 0 to $50 \mu \mathrm{M}$ of compounds $\mathbf{1}$ and $\mathbf{3}$ (a compound was considered active if $\mathrm{IC}_{50} \leq 50 \mu \mathrm{M}$ ). Each compound at each concentration was tested as a triplicate. After $48 \mathrm{~h}$ of incubation, cells were washed and then 3,4,5-dimethylthiazolyl-2-2,5-diphenyltetrazolium bromide (MTT) was added for $4 \mathrm{~h}$. Purple formazan crystals formed were then dissolved in DMSO and the plates were read under $570 \mathrm{~nm}$. $\mathrm{IC}_{50}$ values were determined by non-linear regression. Vincristine was used as a positive control at $\mathrm{IC}_{50}$ determined by MTT assay under the same conditions described above.

\section{Supplementary Information}

Supplementary information is available free of charge at http://jbcs.sbq.org.br as PDF file. 


\section{Acknowledgments}

The authors thank Pontificia Universidad Javeriana (PUJ) and COLCIENCIAS for the financial support through the projects ID 5564 and 120365843351 ; J. E. D. thanks PUJ for the teaching assistantship.

\section{References}

1. Mikhaleva, A. I.; Zaitsev, A. B.; Trofimov, B. A.; Russ. Chem. Rev. 2006, 75, 797; Mikhaleva, A. I.; Zaitsev, A. B.; Trofimov, B. A.; Usp. Khim. 2006, 75, 884.

2. Choi, S.; Lee, J.; Jeong, S.-Y.; Im, I.; Lee, S.-D.; Lee, E.-J.; Lee, S. K.; Kwon, S.-M.; Ahn, S.-G.; Yoon, J.; Han, S.-Y.; Kim, J.; Kim, Y.-C.; J. Med. Chem. 2010, 53, 3696.

3. Lv, X.-H.; Li, Q.-S.; Ren, Z.-L.; Chu, M.-J.; Sun, J.; Zhang, X.; Xing, M.; Zhu, H.-L.; Cao, H.-Q.; Eur. J. Med. Chem. 2016 , 108, 586.

4. Bhandari, K.; Srinivas, N.; Shiva, K.; Shukla, P. K.; Bioorg. Med. Chem. 2015, 23, 6437.

5. Singh, M.; Kaur, M.; Silakari, O.; Eur. J. Med. Chem. 2014, 84, 206.
6. Baker, W.; Harborne, J. B.; Ollis, W. D.; J. Chem. Soc. 1952, 1303.

7. Gulati, K. C.; Ray, J. N.; Curr. Sci. 1936, 5, 75.

8. Meshcheryakova, L. M.; Tsikalova, T. S.; Orlova, E. K.; Burov, Yu. V.; Speranskaya, N. P.; Zagorevskii, V. A.; Khim. Farm. Zh. 1976, 10, 37.

9. Green, J.; Aronov, A.; Pierce, A.; US pat. 7547794 B2 2009 (CA2522595A1).

10. Schann, S.; Stanilas, M.; Morice, C.; Glethlen, B.; US pat. 20120277212 Al 2012 (CA2779073A1).

11. Wang, H.; Xia, Y.; Yang, Z.; Natschke, S.; Lee, K. In Recent Advances in the Discovery and Development of Flavonoids and their Analogues as Antitumor and Anti-HIV Agents; Manthey, J.; Buslig, B., eds.; Springer US: New York, United States, 1998, ch. 14.

12. Ma, X.; Yan, W.; Dai, Z.; Gao, X.; Ma, Y.; Xu, Q.; Jiang, J.; Zhang, S.; Drug Des., Dev. Ther. 2016, 10, 1419.

Submitted: February 13, 2017

Published online: July 5, 2017 